7 BOZLER, C. O., HOLLIS, M. A., NICHOLS, K. B., RABE, S., VERA, A., and CHEN, C. L.: ' $18.5 \mathrm{~dB}$ gain at $18 \mathrm{GHz}$ with a permeable base transistor', IEEE Electron Device Lett., 1985, EDL-6, pp. 456-458

8 TANG, J. Y.: 'Two-dimensional simulation of MODFET and GaAs gate heterojunction FET's', IEEE Trans., 1985, ED-32, pp. 18171823

9 BOZLER, C. O., and ALLEY, G. D.: 'Fabrication and numerical simulation of the permeable base transistor', ibid., 1980, ED-27, pp 1128-1141

10 LIECHTI, C. A.: 'Microwave field-effect transistors-1976', ibid., 1976, MTT-24, pp. 279-300

11 RAVAIOli, U., and FERRY, D. K.: 'MODFET ensemble Monte Carlo model including the quasi-two-dimension electron gas', ibid., 1986, ED-33, pp. $677-680$

\section{INTERFEROMETRIC MEASUREMENTS OF CHROMATIC AND POLARISATION MODE DISPERSION IN HIGHLY BIREFRINGENT SINGLE-MODE FIBRES}

Indexing terms: Optical fibres, Polarisation, Optical measurement, Optical dispersion

Interferometric measurements on highly birefringent singlemode fibres have been performed. The group delay spectrum of each polarisation mode is independently measured over the whole $1.0-1.73 \mu \mathrm{m}$ spectral range. The polarisation mode dispersion is obtained by direct subtraction of the group delay spectra

Increasing interest in coherent optical communication systems and sensor applications in the past few years has led to a great effort in the development of polarisation-preserving singlemode fibres. Correspondingly, a similar effort must be made in the characterisation of the polarisation properties and related parameters of this new class of optical fibres. Several different polarisation-preserving fibres have been developed by enhancing the birefringence of the fibre to a degree high enough to decouple the two polarisation modes over long lengths of fibre. Therefore, among other properties, chromatic dispersion and polarisation mode dispersion (PMD) are of the greatest importance for coherent transmission systems or device applications.

Polarisation mode dispersion at a single wavelength had been measured in highly birefringent fibres by optical short pulse methods, ${ }^{1}$ frequency domain ${ }^{2,3}$ and interferometric techniques. ${ }^{4}$ More recently, the interferometric technique using linear polarisers and a standard scanning Michelson interferometer was extended into the $0.9-1.5 \mu \mathrm{m}$ range by using a white light source and Fourier transform spectroscopy. ${ }^{5}$ In this letter we report simultaneous measurements of chromatic and polarisation mode dispersion of highly birefringent singlemode optical fibres using the interferometric method for group delay measurements in the $1.00-1.73 \mu \mathrm{m}$ spectral range.

The experimental apparatus was a scanning all-fibre Michelson interferometer in which the group delay can be measured by the position of the fringe pattern with an accuracy of $1.6 \mathrm{fs}$. The $\sim 1.85 \mathrm{~m}$-long highly birefringent fibres



Fig. 1 Direct measurement of fringe visibility of high-birefringence fibre at $\lambda=1 \cdot 3 \mu \mathrm{m}$ were inserted into the test fibre arm and the group delay of each polarisation mode was compared to the group delay in the reference arm of the interferometer. Unpolarised light from a halogen lamp was scanned by a monochromator in the full $1.00-1.73 \mu \mathrm{m}$ spectral range with an $8 \mathrm{~nm}$ spectral width.

Fig. 1 shows the direct measurement of the fringe contrast at $\lambda=1.30 \mu \mathrm{m}$ of a highly birefringent fibre. Owing to the different group velocities of each polarisation mode, the fringe pattern presents a double structure, each one corresponding to one polarisation mode. The relative intensity of the two peaks was found to be dependent on the orientation of the axes of the test fibre at the butt coupling to the interferometer, as well as on the residual birefringence of the fibre components of the interferometer. A comparison between the results for different butt coupling conditions showed, however, that the residual birefringence is negligibly small compared to that of the highly birefringent fibre.

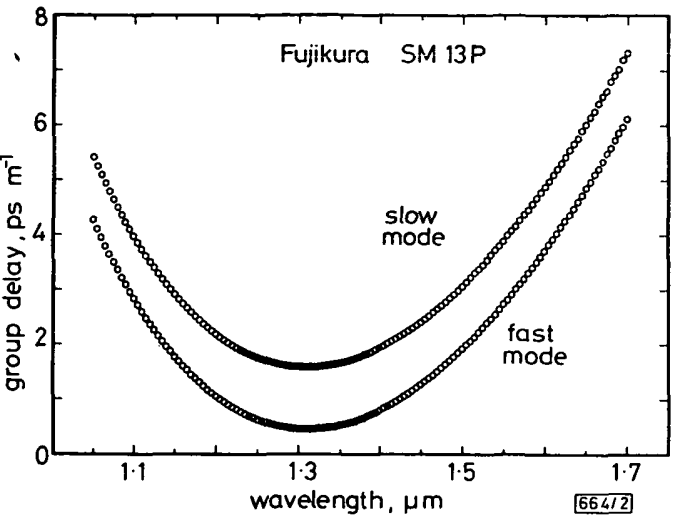

Fig. 2 Grop delay spectra of two polarisation modes of a highbirefringence fibre

Group delay measurements were carried on each $5 \mathrm{~nm}$ in the full spectral range of our apparatus for each polarisation mode. The corresponding results for the Fujikura SM13P fibre are displayed in Fig. 2. The group delay curves were fitted by a five-term Sellmeier expression and the chromatic dispersion of each polarisation mode obtained by simple differentiation of the fitted expression. The polarisation mode dispersion (PMD) at each wavelength was directly obtained by subtraction of the group delay data for each polarisation mode. Typical experimental results are shown in Fig. 3, together with the difference between the two five-term Sellmeier expressions fitting the group delays of each polarisation mode.

The computed results of dispersion measurements of different kinds of polarisation-preserving single-mode fibres are shown in Table 1, together with other measured parameters of the fibres used in the experiments. Fibres A and B present a strong index difference $\Delta n$ and small core dimension. Despite the importance of the waveguide dispersion in the behaviour of these fibres, and the observed core ellipticity of fibres B and $\mathrm{C}$, their PMD was found to be only slightly dependent on the wavelength. In this case, the wavelength-dependent geometrically induced birefringence is much smaller than the stressinduced birefringence, ${ }^{6}$ so the resulting wavelength dependence is comparable to that of fibre $\mathrm{A}$, which presents no core ellipticity.

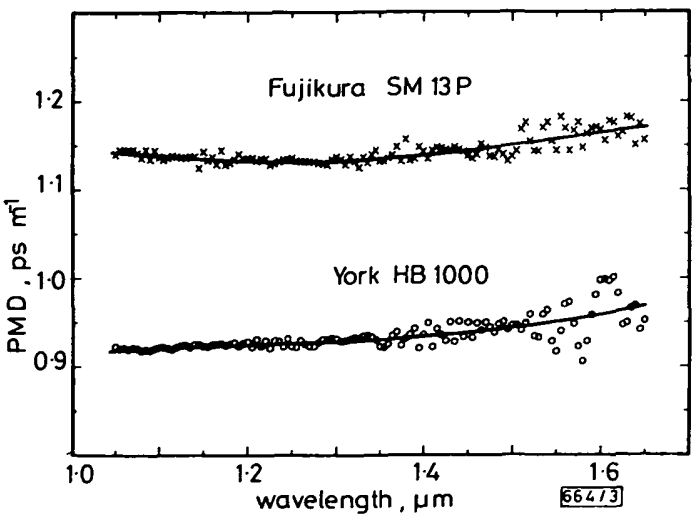

Fig. 3 Polarisation mode dispersion of two different stress-induced highbirefringence fibres 
The minimum PMD which can be accurately measured with this system is limited by the overlap between the fringe pattern of the two polarisation modes. The coherence length of the light source, which defines the spread of the fringe pattern, depends on the wavelength and spectral width of the monochromator as $\lambda^{2} / \Delta \lambda$. On the other hand, the separation of the fringe pattern of the two polarisation modes is proportional to the test fibre length. Therefore, for $\mathrm{a} \sim 1.8 \mathrm{~m}$-long test fibre and an $8 \mathrm{~nm}$-width source at $1.3 \mu \mathrm{m}$ the central peaks of the fringe patterns of the two polarisation modes will start to overlap for fibres with $P M D \leq 0.4 \mathrm{ps} / \mathrm{m}$. Smaller PMDs are better measured with longer samples or widerspectral-width sources rather than introducing a theoretical treatment of the overlap between the two fringe patterns. In this latter case, knowledge of the phase relationship between the two modes is required for correct treatment of the total signal.

Table 1 CHARACTERISTICS OF MEASURED FIBRES

\begin{tabular}{lccc}
\hline & A (Fujikura) & B (York) & C (Corning) \\
\hline $\begin{array}{l}\text { Mean core dia., } \\
\mu \mathrm{m}\end{array}$ & 8.9 & 4.5 & 4.2 \\
Ellipticity & 0 & 0.18 & 0.07 \\
$\Delta n\left(10^{-3}\right)$ & 4.8 & 7.5 & 8.5 \\
PMD at $1.3 \mu \mathrm{m}$, & 1.13 & 0.93 & 1.66 \\
$\quad$ ps/m & 1309.9 & 1397.1 & 1531 \\
$\begin{array}{c}\text { Zero dispersion } \\
\text { slow mode, nm }\end{array}$ & 1310.3 & 1398.4 & 1534 \\
$\begin{array}{c}\text { Zero dispersion } \\
\text { fast mode, nm }\end{array}$ & & & \\
\hline
\end{tabular}

The resolution of the PMD measurements is also limited by the overlap of the wings of the two mode patterns. In the nonoverlapping regime, resolutions of $0.02 \mathrm{ps} / \mathrm{m}$ can be achieved, decreasing to about $0.1 \mathrm{ps} / \mathrm{m}$ before the overlap of the fringe patterns becomes too important for an accurate measurement of the PMD. The effect of this overlap can be observed in the scattering of the experimental points in Fig. 3 above $1.5 \mu \mathrm{m}$, owing to the increasing coherence length of the light source.

In conclusion, the chromatic and polarisation mode dispersion of different types of highly birefringent fibres were measured as a function of the wavelength in the $1.00-1.73 \mu \mathrm{m}$ spectral range. The method is based on the accurate measurement of the group delay for each polarisation mode, without the need for polarising elements in the optical circuit. The main limitation to the method is the overlap between the two mode fringe patterns, so the spectral width of the source and the length of the test fibre must be chosen to avoid such an overlap. The same method was also used in the measurement of the group delay between the fundamental mode and higher modes in the few-mode regime of a single-mode fibre below the cutoff condition.

Acknowledgments: The authors are grateful to H. R. Haller of Swiss PTT and to B. Zysset of the University of Bern for kindly supplying fibre samples for the experiments.

\section{J.-P. VON DER WEID* \\ L. THEVENAZ}

8th December 1986

Group of Applied Physics

University of Geneva

24 Quai Ernest-Ansermet

1211 Geneva 4, Switzerland

\section{J.-P. PELLAUX}

Alphatronix $S A$

13 Route de Chancy

1213 Geneva, Switzerland

* On leave from the Physics Department, PUC/RJ, CP 38075, Rio de Janeiro 22452, Brazil

\section{References}

1 SASAKI, Y., SHIBATA, N., and NODA, J.: 'Splicing of single-polarisation fibres by an optical short-pulse method', Electron. Lett., 1982, 18, pp. 997-999
2 MONERIE, M., LAMOULER, P., and JEUNHOMME, L.: 'Polarisation-mode dispersion measurements in long single-mode fibres', ibid., 1980 16, pp. 907-908

3 YAMABAYASHI, Y., and SARUWATARI, M.: 'New measurement method for polarisation dispersion in single-mode fibres employing frequency-modulated optical signal', ibid., 1983, 19, pp. 239-240

4 MOCHIZUKI, K., NAMIHIRA, Y., and WAKABAYASHI, H.: 'Polarisation mode dispersion measurements in long single mode fibres', ibid., 1981, 17, pp. 153-154

5 EDGE, C., STEWART, W. J., and BENNION, 1.: 'Interferometric measurements of polarization mode dispersion of single-mode optical fibres'. Proceedings of 12th European conference on optical communication, Barcelona, 1986, pp. 271-274

6 RASHLEIGH, S. C.: 'Origins and control of polarization effects in single-mode fibres', J. Lightwave Technol., 1983, LT-1, pp. 312-331

\section{OPTICALLY EXCITED RESONANT DIAPHRAGM PRESSURE SENSOR}

\author{
Indexing terms: Measurement, Optical measurement, Optical \\ sensors \\ Silicon diaphragms fabricated by anisotropic etching are \\ coated with a thin layer of aluminium. An intensity- \\ modulated laser beam focused on the diphragm generates \\ transverse vibrations which are detected interferometrically. \\ Differential pressure is applied to the diaphragm and the \\ pressure-induced change in the fundamental resonant fre- \\ quency is reported.
}

Introduction: Recently, great interest has been shown in a new class of optical microsensors whose output is a frequency which varies as a function of the measurand. A frequencybased sensor of this kind is recognised as a resonant sensor. ${ }^{1}$ In the optical version, the resonant element is directly excited into motion using an intensity-modulated laser beam, and the vibrations are also detected optically. ${ }^{2}$ An all-optical approach, using fibre optics for light transmission, offers the advantage of intrinsic safety and freedom from electromagnetic interference. The output of the sensor system, being a frequency, is readily adapted for digital processing and is transmission-line-independent. A number of optically excited resonant structures have already been reported, ${ }^{3}$ including a resonant diaphragm. ${ }^{4}$ This letter presents the first experimental measurements of the pressure-induced change in resonant frequency of a similar diaphragm.

Experiment: Square diaphragms of sides $750 \mu \mathrm{m}$ and thickness about $5 \mu \mathrm{m}$ are anisotropically etched from single-crystal silicon. An aluminium film of about $0.5 \mu \mathrm{m}$ thickness is evaporated on to one side of the diaphragm. The experimental apparatus used for the investigation of this diaphragm is shown in Fig. 1. Photothermal excitation of the diaphragm is achieved by an intensity-modulated semiconductor laser at a wavelength of $780 \mu \mathrm{m}$. The laser diode is modulated over a range of frequencies up to $1 \mathrm{MHz}$. An average power of $1 \mathrm{~mW}$, with a modulation index of 0.6 , is incident on the diaphragm. Optical detection of the diaphragm deflection is achieved by means of a heterodyne interferometer with an He-Ne laser source. Pressure, either positive or negative with respect to the atmosphere, can be applied to the diaphragm, which is

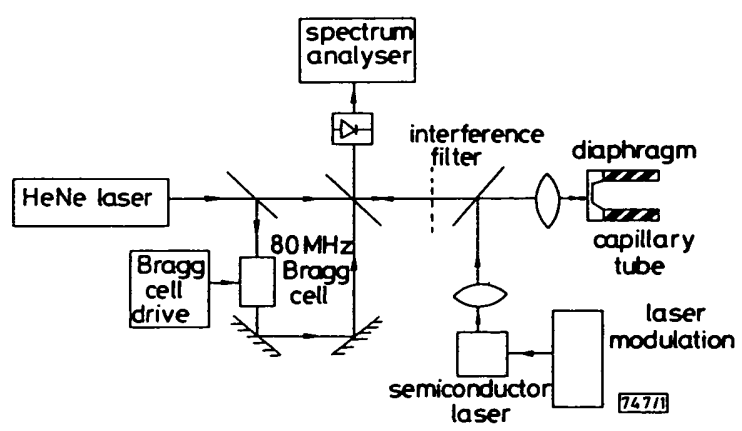

Fig. 1 Experimental arrangement to measure pressure dependence of resonant frequency 